\title{
Impacto de la productividad y eficiencia técnica de los granos básicos en Nicaragua, $1961-2013$
}

\section{Impact of productivity and technical efficiency of basic grains in Nicaragua, $1961-2013$}

\author{
López-González, Álvaro; Zúniga-González, Carlos A.; Rangel-Cura, \\ Raúl Alberto; Dios-Palamares, Rafaela; Colón-García, Adelfa Patricia; \\ Quiros-Madrigal, Olman José; Editor Académico Dr. Ángel Sol \\ Sánchez
}

\author{
Álvaro López-González \\ alvaro_lopez67@yahoo.es \\ Universidad Nacional Autónoma de Nicaragua, \\ Managua. FAREM Carazo, Nicaragua \\ (iD) Carlos A. Zúniga-González \\ czuniga@ct.unanleon.edu.ni \\ Universidad Nacional Autónoma de Nicaragua, León. \\ Escuela de Ciencias Agrarias y Veterinarias., Nicaragua \\ Raúl Alberto Rangel-Cura \\ raulr@geotech.cu \\ Instituto de Geografía Tropical, Cuba., Cuba \\ Rafaela Dios-Palamares \\ ma1dipar@uco.es \\ Universidad de Córdoba. EFIUCO., Spain \\ iD Adelfa Patricia Colón-García \\ pttycolon@gmail.com \\ Universidad Nacional Autónoma de Honduras. \\ CURLA, Honduras \\ (iD) Olman José Quiros-Madrigal \\ olman.quiros@ucr.ac.cr \\ Universidad de Costa Rica. CIEDA, Costa Rica \\ Editor Académico Dr. Ángel Sol Sánchez \\ Colegio de Postgraduados, México, Mexico
}

Revista Iberoamericana de Bioeconomía y Cambio Climático

Universidad Nacional Autónoma de Nicaragua, León, Nicaragua ISSN-e: 2410-7980

Periodicidad: Semestral

vol. 2, núm. 1, 2016

czuniga@ct.unanleon.edu.ni

Recepción: 01 Febrero 2016

Aprobación: 01 Junio 2016

URL: http://portal.amelica.org/ameli/journal/394/3941750003/

DOI: https://doi.org/10.5377/ribcc.v2i1.5682

Autor de correspondencia: alvaro_lopez67@yahoo.es
Resumen: El presente estudio se centró en medir el impacto de las tecnologías implementadas en los granos básicos de Nicaragua para mejorar la productividad total de los factores (PTF), considerando la variabilidad del clima. Se utilizó la base de datos de la FAO para los rubros: Arroz, maíz y frijol. Se aplicó la metodología de los índices de Malmquist, que mide la productividad total de los factores y explica las causas de estas en bases a la Tecnología y la Eficiencia Técnica. Esta a su vez es explicada por la Eficiencia a escala y la Eficiencia pura. Los resultados evidencian un comportamiento no uniforme del indicador PTF para cada uno de los rubros estudiados (arroz, frijol y maíz) durante el período 1961 - 2013. Además se observa que la evolución de los indicadores obtenidos se explica a partir de los indicadores de cambio tecnológico con poca incidencia del indicador de eficiencia técnica en tal comportamiento. El cultivo del frijol es el rubro que presenta una mayor evolución en su indicador PTF con un 12\%, seguido del cultivo del arroz con un $11 \%$ y el cultivo del maíz con un $5 \%$.

Palabras clave: Productividad, Eficiencia técnica, Tecnología, Eficiencia a Escala, Eficiencia pura.

Abstract: This study focused on measuring the impact of the technologies implemented in basic grains in Nicaragua to improve total factor productivity (TFP), considering climate variability. The FAO database was used for the items: Rice, corn and beans. The methodology of the Malmquist indices was applied, which measures the total productivity of the factors and explains the causes of these based on Technology and Technical Efficiency. This in turn is explained by Efficiency at scale and Pure Efficiency. The results show a non-uniform behavior of the TFP indicator for each of the items studied (rice, beans and corn) during the period 1961 - 2013. It is also observed that the evolution of the indicators obtained is explained from the indicators of change technology with little incidence of the indicator of technical efficiency in such behavior. Bean cultivation is the item that shows the greatest evolution in its TFP indicator with $12 \%$, followed by rice cultivation with $11 \%$ and corn cultivation with $5 \%$. 
Keywords: Productivity, Technical efficiency, Scale Efficiency, Pure Efficiency.

\section{INTRODUCCIÓN}

La escasez de recursos hace necesaria la eficiencia en la producción, (Martínez et al 2013) sobre en todo en países en vías de desarrollo donde en muchos casos abundan los recursos naturales pero son limitados los recursos tecnológicos. El crecimiento productivo en el sector agrícola es considerado esencial, es decir si los productos del sector agrícola crecen rápidamente como para satisfacer la demanda de alimentos en relación al crecimiento poblacional (Zúniga y Navarrete 2011b). Este concepto, de eficiencia productiva, denota un carácter comparativo y no absoluto y permite comparar los niveles productivos en una línea de tiempo definida. Considerando las características de esta magnitud económica se presenta un estudio de la PTF de tres rubros agrícolas fundamentales en la economía nicaragüense: maíz, arroz y frijoles.

En este artículo se emplea el método de análisis de datos envolventes con el Índice de Malmquist. Este índice evalúa cada rubro referente al grupo en dos puntos del tiempo, generando una medida de eficiencia del rubro bajo análisis. Con datos de Producción y rendimientos físicos por hectárea, utilización de semillas, mano de obra y variaciones climáticas de aproximadamente los últimos 50 años.

Generalmente, se acepta que unidades productivas que con los mismos insumos obtenga más producto, o que para la misma cantidad de producto obtenido emplee menos insumos, sean las más eficientes. En Nicaragua se tiene información de la producción de gran parte de los rubros agropecuarios sobre todo de granos básicos y dada la escasez de recursos, es pertinente un estudio de la Productividad Total de los Factores en todos los sectores, esencialmente de los sectores generadores de materia prima, alimentos y empleo.

En este estudio, se tiene como base de datos las estadísticas de la FAO y el Banco Central de Nicaragua, se analizo el comportamiento de tres rubros agrícolas, que pueden representar el punto de partida para posteriores estudio, y se hace énfasis en la productividad de los factores tierra, semilla y mano de obra y se toma en consideración el factor de variabilidad climática experimentado durante el período de estudio (1961 - 2013).

Para el estudio empírico de esta temática es necesario contar con estimaciones de los cambios en la Productividad global o Productividad Tota de los Factores, definida esta como un residuo entre el cambio esperado del producto y en los insumos y calculada a través de números índices que agregan en forma adecuada la producción de distintos bienes y los insumos necesarios para producirlos (Arimón y Torrelo 1997).

En el ámbito rural nicaragüense la agricultura, la ganadería y la forestaría son las actividades que generan mayor empleo e ingreso, además tienen gran potencial de complementar servicios como agroturismo y turismo rural entre otros. Dentro de la producción agrícola se encuentra una gran diversidad de rubros, que tradicionalmente se dividen en productos de consumo interno, principalmente granos básicos y productos de exportación, concentrados principalmente en seis productos. Los granos básicos en su conjunto (arroz oro, frijol, maíz y sorgo) en promedio participan en casi el $40 \%$ del PIB agrícola. De estos rubros el que más aporta es el frijol con 13\%, seguido del arroz con casi el 11\% del PIB agrícola. La mayoría de los rubros de consumo interno han experimentado crecimiento destacándose el arroz (de riego y secano), el maíz y el frijol, debido tanto a incrementos en su rendimiento por manzana, como al total del área sembrada (FUNICA 2012).

Lo anterior destaca la importancia económica de estos cultivos, no obstante se aclara que los incrementos en la producción que se han observado, se deben mayormente al crecimiento de las áreas de siembra y en

\section{NotAS DE AUTOR}


menor medida a la productividad. Los cuadros siguientes muestran el comportamiento de estos rubros en el período 1961 - 2014:

En el gráfico 1 se presenta la evolución de área cosechadas de los rubros estudiados medidos en Hectáreas (Ha), se puede notar el en Nicaragua el rubro maíz es el que ha tenido mayor cobertura seguido del frijol seco

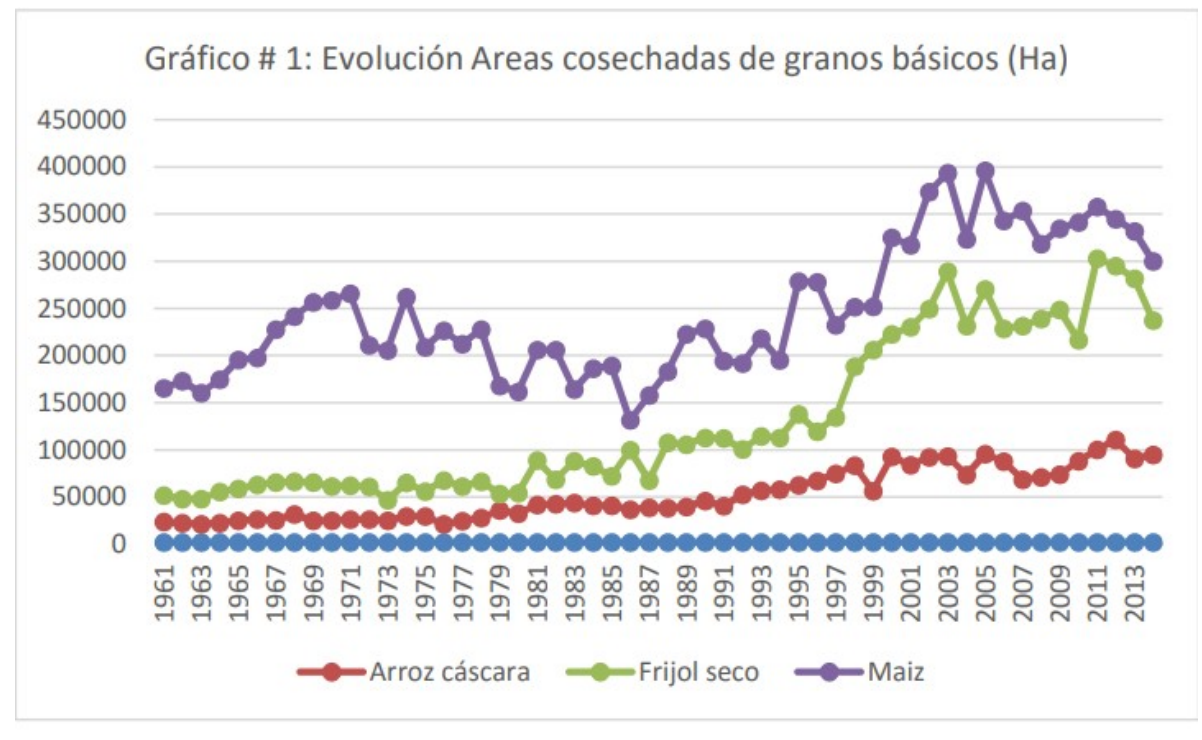

GRÁFICO 1

Evolución de área cosechadas

En el gráfico 2 se presenta la evolución de la producción de los rubros estudiados medidos en Toneladas $(\mathrm{Tn})$, en orden ascendente se nota que la producción de maíz y arroz se destacan por su mayor volumen.

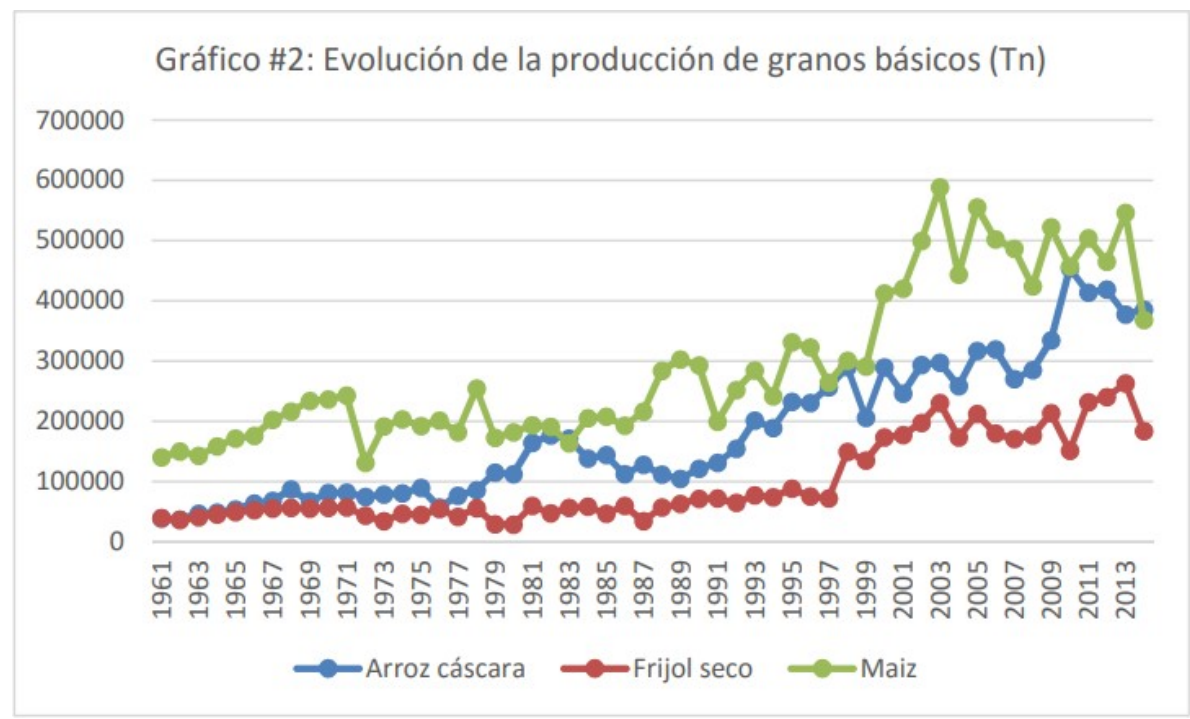

GRÁFICO 2

Evolución de la producción

En la gráfica 3 se presenta la evolución de los rendimientos de los rubros estudiados, de tal manera que de mayor rendimiento el arroz seguido del maíz. 


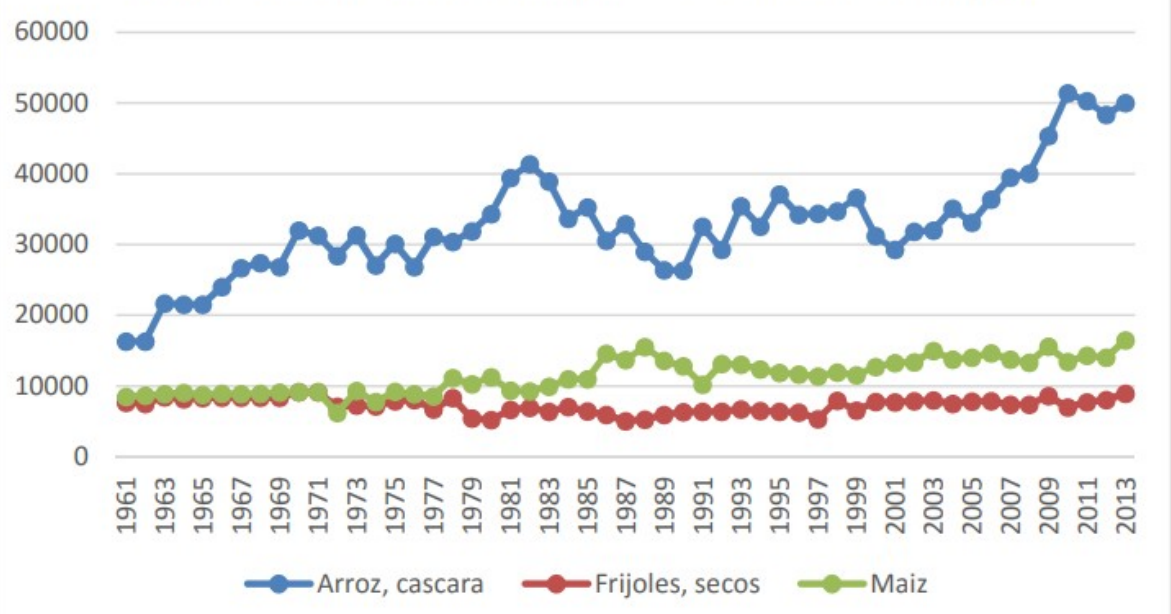

\section{REVISión DE LA LiTERATURA}

Saini \& Lema(2015) indican que la mayor parte de los estudios previos muestran que en general se han aplicado cuatro métodos principales para la estimación: a) Modelos econométricos, b) índice de productividad Total de los factores (PTF) (Tornqvist/Fisher/Malmquist), c) Análisis Envolvente de Datos (DEA); y d) fronteras estocásticas (SF). Tabla 1

TABLA 1

Resumen de ventajas y desventajas de metodos

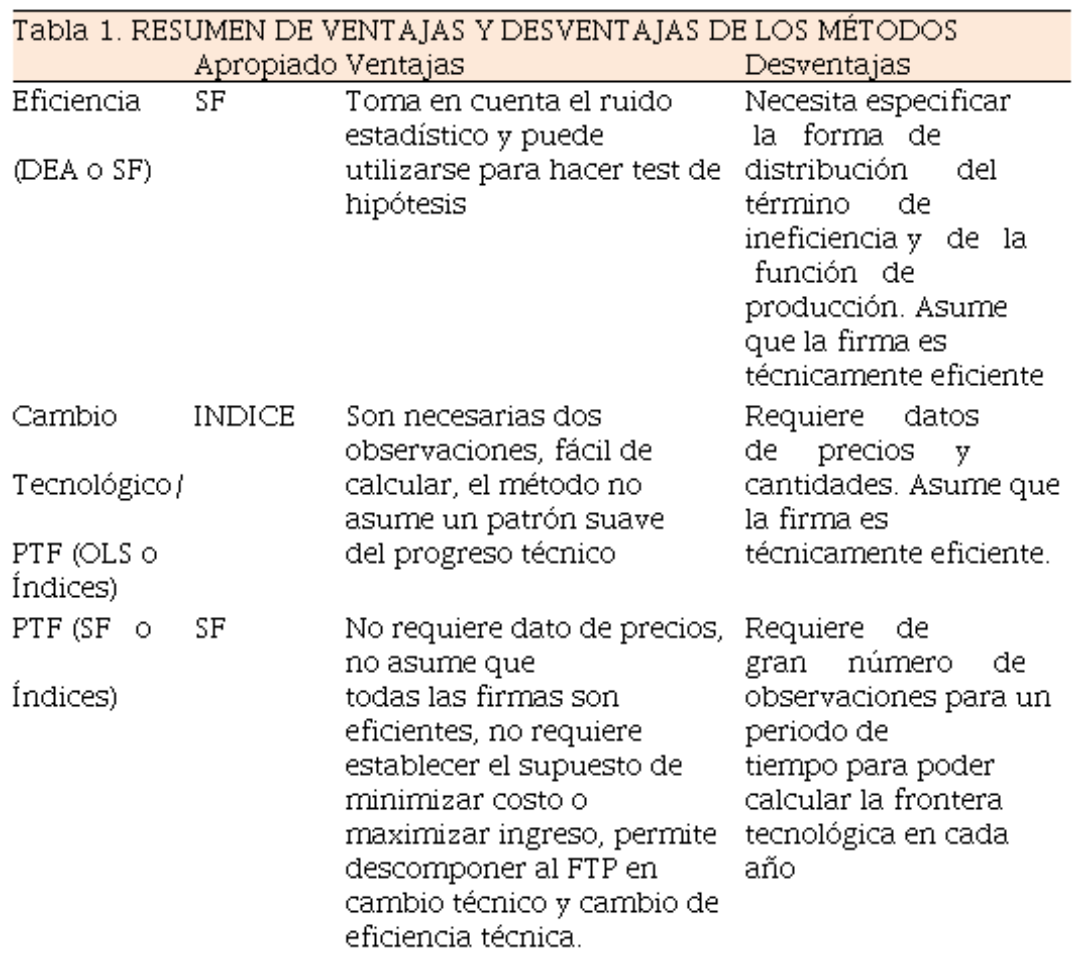


En nuestro estudio se hace uso de los índices Malmquist, los cuales permiten descomponer los cambios en la productividad, en cambios en la eficiencia y en cambios en la tecnología. Para determinar la distancia entre los puntos de producción y la frontera tecnológica, se emplea, en el trabajo, una versión de la metodología DEA (con orientación hacia el producto), bajo retornos constantes a escala, que involucra métodos no paramétricos de programación. Esta metodología hace posible estimar los índices Malmquist de cambios en la productividad, a través del tiempo.

Lanteri (2002) señala que los índices Malmquist fueron introducidos originalmente en el ámbito de la teoría del consumo (Malmquist, 1953). Esta propuesta fue posteriormente aplicada a la medición de la productividad, por Caves, Christensen y Diewert (1982), en un contexto de funciones de producción, y por Fare, Grosskopf, Lindgren y Roos (1989), en un contexto (DEA) no paramétrico. Los índices de productividad Malmquist han sido aplicados en varios estudios sobre productividad en países de nuestro hemisferio, entre ellos Olavaria (2005), Zúniga- González (2011a,b,c), Araujo et. al (2014), Lema, Gatti, \& Brescia (2015).

Para definir los índices Malmquist de cambios en la productividad (orientados hacia el producto) se supone que, para cada período de tiempo $t=1, . ., \mathrm{T}$, la tecnología $\mathrm{St}$ (un conjunto no vacío, cerrado y convexo) permite transformar $\mathrm{xt}$ insumos en yt productos, donde : (Lanteri 2002) define ec 1.

$$
S_{t} \square\left(x \square_{t} \downarrow, y_{t}\right): x_{t} \text { paraproducir } \mid-y_{t}
$$

Shephard (1970) define la función distancia producto en t como:ec 2

$$
\begin{gathered}
\text { D_t } 0 \text { (x_t,y_t }) \\
\square \inf \square \phi \dashv:\left(x_{t}, \square \frac{y_{t}}{\phi} \square\right) \square+S_{t}=\left(\sup \phi \dashv:\left(x_{t}, \phi y_{t}\right) \square \mid-S_{t}\right)-1
\end{gathered}
$$

Para calcular los índices Malmquist es necesario definir funciones distancia con respecto a dos períodos de tiempo diferentes ec 3 :

$$
D_{t} 0\left(x_{t}+1, y_{t}+1\right)=\square \inf \square \phi \dashv:\left(x_{t}+1, \square\left(y_{t}+1 \frac{)}{\phi} \square\right) \square-S_{t}\right.
$$

Bravo Uretra, citando a Russel (1983) asegura que para lograr un aumento significativo en la productividad y la competitividad, se requiere de políticas que fomenten la adopción de nuevas tecnologías, así como medidas que promuevan el uso eficiente de las tecnologías existentes. Además, señala, que es necesario que los responsables políticos, productores y extensionistas agrícolas tengan acceso a los estudios empíricos que les permitan dilucidar los efectos de los diferentes factores que influyen en los aumentos de la productividad.

No obstante, y a pesar de la importancia y la urgencia de abordar esta temática hay pocos estudios especializados que tengan en cuenta el comportamiento de las variables de productividad de sectores tan importantes como el agrícola y pecuario en los países del área.

Y es que la vertiginosa liberalización de los mercados, acelerada por la firma de innumerables tratados de libre comercio en las últimas décadas, ha potenciado la amenaza de la competencia de productos importados frente a productos locales. Amenaza que solo podrá enfrentarse si los diferentes sectores económicos, esencialmente los sectores agrícolas y pecuarios, mejoran sustancialmente el uso eficiente de los recursos con que cuentan. 
Bravo Uretra (2008a,b) afirma que existen varios factores, como aumentos en los ingresos y en la población, variabilidad climática, incrementos en los precios de alimentos destinados a energéticos, y los procesos de globalización y urbanización que están transformando los mercados y llevando a alzas significativas en los precios de granos básicos y alimentos en general. Citando a Runge y Senauer, (2007) señala: "evidentemente, esta situación puede ser muy complicada en los países más pobres donde el incremento en el costo de alimentos tiene un gran efecto en los ingresos reales e incluso en la estabilidad política." Además añade que "En un análisis reciente, Von Braun (2007) sugiere varias estrategias para paliar esta situación. Una de ellas, de particular relevancia, es la necesidad de invertir en investigación para desarrollar y difundir nuevas tecnologías que lleven a aumentos en la productividad de la agricultura campesina".

La evidencia empírica indica que en regiones o países que no se han beneficiado del crecimiento de la productividad en la agricultura, los agricultores han perdido ventaja competitiva. Es más, desde una perspectiva macroeconómica, el crecimiento de la productividad en la agricultura contribuye a la reducción en el precio de los alimentos, lo que en países pobres significa un aumento en el poder adquisitivo de los sueldos, bajando así el costo del desarrollo industrial (Ruttan, 2002). LA PRODUCTIVIDAD TOTAL DE LOS FACTORES (PTF) La PTF es un concepto que se vincula directamente con el cambio tecnológico. En términos operativos una medida de la PTF puede ser definida como ec 4:

$$
\mathrm{PTF}=\frac{Y}{X}
$$

donde PTF mide el producto agregado Y, producido por unidad del insumo agregado X. En consecuencia la tasa de crecimiento de la productividad será la tasa de crecimiento del producto menos la tasa de crecimiento de los insumos.

En la práctica, todo el mundo calcula el progreso técnico siguiendo el método que Solow planteó hace más de 50 años para establecer las fuentes del crecimiento económico, y muchos autores citan su propuesta en los trabajos que se han escrito sobre cambio técnico.

Solow (1957) publicó su artículo sobre el cambio tecnológico y la función de producción agregada en 1957. Desde entonces, el procedimiento para distinguir entre las variaciones de la producción debidas al progreso técnico y a los cambios en la disponibilidad de

capital ha tenido sustento teórico en su trabajo seminal. Su contribución consiste en cuantificar el progreso técnico en forma residual. Por esta razón, al progreso técnico se lo conoce también como residuo de Solow o productividad total de los factores (PTF).

Los fundamentos teóricos del progreso técnico se pueden representar en forma matemática y gráfica. Matemáticamente, la función de producción agregada de la economía se puede escribir así: ec 5

$$
Y=f(K, L, t)
$$

donde $\mathrm{Y}$ es el producto de la economía, K el capital utilizado resultante de los aumentos sucesivos de la inversión y t el progreso tecnológico que experimenta la economía a través del tiempo, y que refleja cualquier desplazamiento de la función.

Se puede plantear, así mismo, una función de producción explícita como la Cobb- Douglas, que tiene la propiedad de ser homogénea de grado uno.

Formalmente se tiene: ecuacion 6

$$
Y=\mathrm{AK} \alpha \mathrm{L} \beta
$$


Expresando esta función como el producto por trabajador en función del capital por trabajador se llega a la siguiente ecuación: yp = Akap donde yp es el producto por trabajador, A el progreso técnico, kp la relación capital trabajo y $\alpha$ sigue siendo la participación de la remuneración del capital dentro del producto.

Ludema (2010) afirma que crecimiento de la productividad en la agricultura ha captado el interés de los economistas desde hace mucho tiempo. A medida que se desarrolla la agricultura, libera recursos para otros sectores de la economía. Esta ha sido la base del éxito de la industrialización en las economías desarrolladas. Por lo tanto, el desarrollo agrícola se convierte en un requisito importante de la transformación estructural hacia el desarrollo industrial, ya que precede y promueve la industrialización.

Haciendo uso de diversos enfoques se ha calculado la PTF para algunos países del hemisferio Chile, Argentina (Olavaria, 2005. Lema et al., 2015) y en algunos casos para la totalidad de los países de América Latina y el Caribe (Ludema, 2010 y A Araujo et al., 2014).

Olavaria (2005) en su estudio Productividad Total de los Factores en la agricultura chilena: 1961 - 1996 tomó como referencia estudios anteriores los que en su mayoría utilizaron el índice de Malmquist para calcular la PTF en este país andino. Tabla 2

TABLA 2

Estudios Previos sobre PTF en el sector agrícola chileno

\begin{tabular}{|c|c|c|c|}
\hline Trueblood y Coggins & 1961 & Índice de & $1.40 \%$ \\
\hline (2003) & -1991 & Malmsquist & \\
\hline Coelli y Prasada Rao 200 & $\begin{array}{l}51980- \\
2000\end{array}$ & $\begin{array}{l}\text { Índice de } \\
\text { Malmsquist }\end{array}$ & $1.10 \%$ \\
\hline $\begin{array}{l}\text { Nin, Arndt y Preckel } \\
(2003)\end{array}$ & $\begin{array}{l}1961- \\
1994\end{array}$ & $\begin{array}{l}\text { Índice de } \\
\text { Malmsquist }\end{array}$ & $0,9 \%$ \\
\hline Martin y & $1967-$ & Translog y Cobb & $2.4 \%-2.7 \%$ \\
\hline $\begin{array}{l}\text { Mitra } \\
(1999)\end{array}$ & 1992 & Douglas & \\
\hline $\begin{array}{l}\text { Arnade } \\
(1998)\end{array}$ & $\begin{array}{l}1961- \\
1993\end{array}$ & $\begin{array}{l}\text { Índice de } \\
\text { Malmsquist }\end{array}$ & $1.30 \%$ \\
\hline Fulginiti y & $1961-$ & Índice de & $1.1 \%-0.8 \%$ \\
\hline $\begin{array}{l}\text { Perrin } \\
(1997)\end{array}$ & 1985 & Malmsquist & \\
\hline
\end{tabular}

Autores Período Metodología Cambio promedio

Fuente Elaboracion propia

Lema, et al (2015) , Lema, Brescia (2001) para calcular la PTF en Argentina también hizo referencia a estudios anteriores, lo que evidencia al igual que en Chile la pertinencia del abordaje del tema de la PTF y la identificación delos factores claves en su comportamiento. Tabla 3

TABLA 3

Resumen de diversas estimaciones de la PTF del sector agrícola que incluyen a Argentina

\begin{tabular}{|c|c|c|c|c|}
\hline Arnade & (1998) & $\begin{array}{l}1960- \\
1993\end{array}$ & DEA & $1.90 \%$ \\
\hline Fulginiti y & (1998) & $1961-$ & DEA & $4.80 \%$ \\
\hline Perrin & & 1985 & & \\
\hline Lema & (1999) & $\begin{array}{l}1970- \\
1997\end{array}$ & $\begin{array}{l}\text { Relaciones } \\
\text { contables }\end{array}$ & $1.55 \%$ \\
\hline \multicolumn{2}{|c|}{$\begin{array}{l}\text { Artana, Cristini y } \\
\text { Pantano }\end{array}$} & & $\begin{array}{l}\text { Funciones de } \\
\text { producción }\end{array}$ & $2.20 \%$ \\
\hline \multicolumn{2}{|l|}{ Coeeli y } & $1980-$ & Malmsquist y DEA & $2.70 \%$ \\
\hline \multicolumn{2}{|l|}{$\begin{array}{l}\text { Rao } \\
(2005)\end{array}$} & 2000 & & \\
\hline \multirow{2}{*}{\multicolumn{2}{|c|}{ Nin, Arndt y }} & $1965-$ & DEA & $2 \%$ \\
\hline & & 1994 & & \\
\hline
\end{tabular}


La productividad agrícola juega un papel clave en el proceso de industrialización y desarrollo. Algunos autores muestran que los países con altos niveles de crecimiento de la productividad han sido exitosos en sus procesos de industrialización. Mientras tanto, los países con bajos niveles de crecimiento de la productividady un fuerte sesgo en contra de la agricultura a través de las políticas comerciales y de precios fueron infructuosos en sus fases de industrialización, Nin:Prat et al., (2015).

Ludema (2010), en su estudio para el BID en el año 2010 señala que en América Latina y el Caribe , la mayor parte del análisis del crecimiento de la productividad total de los factores (PTF) en la agricultura en los últimos 20 años ha sido en el contexto de los estudios de múltiples países de todo el mundo y señala los estudios realizados por Fulginiti y Perrin , (1993), por Arnade , 1998; Trueblood y Coggins , 2003; Nin , Arndt y Preckel , 2003; Coelli y Rao , 2005; . Wiebe et al, 2000; Bravo, Ortega y Lederman, 2004; Ludema et al., 2007). Estos estudios, señala Ludema, ofrecen una visión amplia de crecimiento de la productividad agrícola y presentan los resultados de ciertos países de América Latina.

Andrade Araujo (2014) amplia los alcances de estudios anteriores sobre la PTF en América Latina y el caribe realizando un análisis que va desde el año 1962 hasta el año 2010. Este autor indica que el método utilizado en este trabajo se denomina análisis de frontera de producción estocástica y constituye uno de los métodos adoptados en la literatura sobre ineficiencia técnica. Mediante ese análisis se obtiene uno de los componentes de la PTF denominado eficiencia técnica. En este enfoque se emplean técnicas econométricas (paramétricas), con cuyos modelos de frontera de producción se estudia la ineficiencia técnica y se reconoce que el producto puede resultar afectado por perturbaciones aleatorias, ajenas al control de los productores. Al contrario de los enfoques no paramétricos, que suponen fronteras determinísticas, el análisis de frontera estocástica permite desviaciones de la frontera cuyo error puede descomponerse para distinguir entre alteraciones de eficiencia técnica y perturbaciones aleatorias. Tabla 4

TABLA 4

Resultados de la PTF promedios 1962-2010 países de Centroamérica

\begin{tabular}{lllll} 
Costa Rica & 0,05160 & 0,0049 & 0,0022 & 0,0038 \\
Guatemala & 0,04110 & 0,0051 & 7 & 0,0041 \\
El Salvador & 0,03550 & 0,0101 & 4 & 0,0048 \\
Honduras & 0,04480 & 0,00790 & 0,0006 & 0,0062 \\
Nicaragua & 0,04090 & 0,0077 & 0,0005 & 0,0098 \\
\hline
\end{tabular}

PAIS CREC. ECONOMICO CAMBIO EN PTF PROG. TECNICO EFICI. TECNICA

Zúniga-González $(2011 \mathrm{~b}, \mathrm{c})$ señala que el ritmo de crecimiento de la productividad en los subsistemas de producción dependen de las tecnologías (el cómo hacer) y de la capacidad de la mano de obra (gestión del conocimiento). Por consiguiente, medir la productividad en la economía debería ser un referente en la toma de decisiones para los hacedores de política.

En su trabajo, Zúniga-González (2011a,b), presenta un resumen de indicadores obtenidos por otros autores respectos al comportamiento de la PTF en Nicaragua. Tabla 5 
TABLA 5

Resultados de estudios sobre PTF en el sector agrícola de Nicaragua

\begin{tabular}{|c|c|c|c|c|}
\hline Leudena & $(2010)$ & 2010 & $\begin{array}{l}\text { Malmsquist } \\
\text { DEA }\end{array}$ & $1.40 \%$ \\
\hline Nin y Bingxin & (2008) & $\begin{array}{l}1984- \\
2003\end{array}$ & $\begin{array}{l}\text { Malmsquist } \\
\text { DEA }\end{array}$ & $1,19 \%$ \\
\hline Bravo-Ortega & (2004) & $\begin{array}{l}1960- \\
2000\end{array}$ & Translog & $0.80 \%$ \\
\hline $\begin{array}{l}\text { Avila y } \\
\text { Evenson }\end{array}$ & (2005) & $\begin{array}{l}1961- \\
2004\end{array}$ & OLS & $1.60 \%$ \\
\hline $\begin{array}{l}\text { Coelli y } \\
\text { Prasada }\end{array}$ & (2003) & $\begin{array}{l}1980- \\
2000\end{array}$ & $\begin{array}{l}\text { Malmsquist } \\
\text { DEA }\end{array}$ & $1 \%$ \\
\hline $\begin{array}{l}\text { Trueblood y } \\
\text { Coggins }\end{array}$ & (2003) & $\begin{array}{l}1961- \\
1991\end{array}$ & $\begin{array}{l}\text { Malmsquist } \\
\text { DEA }\end{array}$ & $-3 \%$ \\
\hline Arnade & (1998) & $\begin{array}{l}1961- \\
1993\end{array}$ & $\begin{array}{l}\text { Malmsquist } \\
\text { DEA }\end{array}$ & $-2 \%$ \\
\hline
\end{tabular}

Tomado de Impacto de la política salarial y de empleo en la productividad de los sectores productivos en Nicaragua, 1994-2010, Zúniga-González (2011b).

\section{Datos}

Los datos se organizaron en Panel de datos. El panel incluye los rubros de arroz, maíz, y frijol. Se organizaron en una serie de tiempo 1960-2013. Se consideraron las variables input área, rendimiento, semilla y como variable ambiental Ap. Se utilizó la disponibilidad de datos en la FAO y web del Banco Central de Nicaragua, donde es posible acceder y descargar todos los datos necesarios de la sección de estadísticas.

El estudio incluye los sectores los rubros arroz, maíz, frijol del subsector agrícola de la economía nicaragüense.

Los resultados son presentados para el período de estudio 1960-2013, se excluyen los años (BCN: 2009).

Serie Output (Salida)

En la serie de tiempo Output es designado para la producción de los rubros arroz, maíz y frijol, medida en toneladas. 1 Tonelada equivale a 2000 libras.

Series Inputs (Entradas)

De igual manera, la serie de inputs se organizan en panel de datos por rubros y por años. Input 1: Área cosecha representa el área cosechada de cada rubro estudiado en la serie de tiempo, medido en Hectárea.

Input2: Salario representa el salario promedio de afiliados al INSS según actividad económica, del sector agropecuario de la base de datos del Banco Central de Nicaragua Cuadro - Table III-7, medido en córdobas, comprende el periodo 1960-2013.

Input3: Rendimiento representa la producción entre el área cosechada medido en hectogramo / Ha. Un hectogramo equivale a $01 . \mathrm{Kg}$. La Hectárea equivale a $10,000 \mathrm{~m}^{2}$.

Input4: Semilla corresponde a cada rubro estudiando en la serie de tiempo indicada medida en toneladas.

Input5: (Ap) Representa un índice de la actividad magnética planetaria medida en promedio para el periodo de estudio, medido en ij nanoteslas nT. ). La Actividad Solar y Geomagnética es tenida en cuenta como variable medioambiental con posible influencia En el tema tratado, dados los numerosos reportes que muestran su relación con la biota en general y con la actividad de microorganismos, con la velocidad de reacciones físico- químicas, productividad pesquera y melífera, entre otros procesos biológicos (Aguilar, A. J. et al: 2014).

En la tabla No 6 se presentan la estadística descriptiva de las variables que se organizaron en panel de datos de 1961-2014. Resultando significativo para la aplicación de la herramienta para el análisis de datos envolventes. 


\begin{tabular}{|c|c|c|c|c|c|c|}
\hline Estadísticos & Produccion & Area & Rendimiento & Semilla & Mano de obra & $\begin{array}{c}\text { Radiacion } \\
\text { solar }\end{array}$ \\
\hline Media & 187922.88 & 142819.5741 & 19429.50617 & 5720.117284 & 939265.6185 & 13.28759259 \\
\hline Error típico & 10511.12 & 8071.299232 & 1970.255587 & 417.6739897 & 527896.6357 & 0.344582107 \\
\hline Mediana & 172000.00 & 103215 & 11705 & 3733 & 1533.9 & 12.68 \\
\hline Moda & 48400.00 & 22500 & 8871 & 2400 & 553.8 & 11.98 \\
\hline Desviación estándar & 133784.71 & 102730.8676 & 25077.25955 & 5316.121988 & 6719027.236 & 4.385814202 \\
\hline Varianza de la muestra & 17898347981.71 & 10553631149 & 628868946.6 & 28261152.99 & $4.51453 E+13$ & 19.23536622 \\
\hline Curtosis & 0.35 & -0.791203281 & 71.45750394 & 5.381713124 & 50.57855701 & 0.0044291 \\
\hline Coeficiente de asimetría & 0.97 & 0.625664749 & 7.350140683 & 2.339163799 & 7.20702921 & 0.431934652 \\
\hline Rango & 560204.00 & 375154 & 272820 & 24583 & 49697017 & 19.72 \\
\hline Míni mo & 28395.00 & 21102 & 5035 & 1417 & 492.1 & 3.93 \\
\hline Máximo & 588599.00 & 396256 & 277855 & 26000 & 49697509.1 & 23.65 \\
\hline Suma & 30443506.00 & 23136771 & 3147580 & 926659 & 152161030.2 & 2152.59 \\
\hline Cuenta & 162.00 & 162 & 162 & 162 & 162 & 162 \\
\hline Nivel de confianza(95.0\%) & 20757.44 & 15939.26703 & 3890.876676 & 824.8259745 & 1042494.548 & 0.680483533 \\
\hline
\end{tabular}

\section{Aplicación Empírica:}

Índices de Malmquist

A partir del trabajo pionero de Solow (1957), y Reyez Bernal (2010) sobre la contabilidad del crecimiento (factor residual), ha sido común medir el cambio en la PTF como una forma de buscar las causas del crecimiento y de esta manera reducir lo que se ha dado en llamar la medida de nuestra ignorancia. Los avances en este campo han sido significativos y en un primer momento se han orientado a distinguir los determinantes de la acumulación de factores, tratando de observar tanto la calidad de la mano de obra como del capital. En realidad, se pretendía dar respuesta a las siguientes preguntas: ¿por qué crece una economía? y ¿̇cuál era la posibilidad de convergencia? (Sotelsek y Laborda 2010).

Para estimar la productividad total de los factores en la agricultura se utiliza el Índice de Malmquist (Färe et al., 1994), una metodología no paramétrica que utiliza el análisis envolvente de datos (DEA) métodos para construir una frontera de producción lineal por tramos para cada país y año en la muestra. Esta metodología se ha utilizado ampliamente para la medición de la productividad agrícola, ya que ofrece algunas ventajas.

Este enfoque : i) no requiere información sobre los precios, ii ) no da por sentado que todos los países son eficientes, iii ) no asume una función objetivo de conducta tales como la minimización de costes o de maximización de los ingresos / beneficios, y iv ) permite la descomposición de la PTF en cambio técnico, cambio en la eficiencia y el cambio de escala .(Ludema et al., 2015).

Se generó una base de datos para el análisis de la productividad total de los factores a partir de datos de la FAO; se empleó como variable Producto la producción en quintales por hectárea y como variables insumos: la tierra, las semillas y las condiciones climáticas.

Para el estudio de los índices de productividad basados en funciones distancia se identifican dos enfoques diferenciados. El primer enfoque se inicia con Caves, et al. (1982), que introducen de manera teórica el IM de productividad en insumos y productos. Éste se basa en razones o cocientes con funciones distancia orientadas a insumos y funciones distancia orientadas a productos. La obtención empírica de estos índices pudo realizarse hasta que Färe et al. (1994), mostraron un método de cálculo a través de técnicas de programación lineal (segundo enfoque).

Martínez et al (2013) señala que el Índice de Productividad de Malmquist, representa el crecimiento de la productividad total de los factores (PTF) de una unidad productiva. Refleja el progreso en eficiencia de conformidad con los cambios tecnológicos en el tiempo, que se manifiesta como desplazamiento de la propia frontera bajo un marco de múltiples insumos y productos (Caves et al., 1982). Bajo este enfoque, las observaciones fuera de la frontera reflejan los períodos en los cuales la utilización de los recursos resulta menos eficiente en comparación con las prácticas empleadas durante los mejores años. La distancia entre la frontera y los puntos de producción representa la ineficiencia técnica. Las funciones distancia o medidas de eficiencia 
técnica, permiten cuantificar la ineficiencia técnica en un plan de producción, mediante la comparación de las cantidades de insumos y de productos que necesita una unidad que es técnicamente eficiente con los que utiliza una unidad ineficiente.

Para la obtención de los indicadores de PTF se utilizó un software denominado DEAP en su versión 2:1 que permite la obtención de Índices de Malmquist haciendo usos de un panel de datos mediante el enfoque del Análisis Envolvente de Datos (DEA). Este programa computacional ha sido usado en estudios similares en América Latina y otras partes del mundo y fue desarrollado por el Doctor Tim J. Coelli (2008) del Centro para el análisis de la productividad y la eficiencia del departamento de Econometría de la Universidad de New England, Australia.

\section{Resultados y discusión}

El propósito de nuestra investigación se centró en medir el impacto de las tecnologías implementadas por los productores/as en la producción de granos básicos en Nicaragua para mejorar la productividad total de los factores, en condiciones de variaciones climáticas.

La tabla 8 muestra el promedio de los cambios en la eficiencia técnica, la tecnología, la eficiencia pura, la eficiencia a escala y la productividad total de los factores, durante el período $1961-2013$.

\begin{tabular}{lcccll} 
& $\begin{array}{l}\text { Cambio en } \\
\text { la eficiencia } \\
\text { Récnica }\end{array}$ & $\begin{array}{l}\text { Cambio en } \\
\text { la }\end{array}$ & $\begin{array}{l}\text { Cambio en } \\
\text { la eficiencia } \\
\text { técnica } \\
\text { pura }\end{array}$ & $\begin{array}{l}\text { Cambio en } \\
\text { la eficiencia } \\
\text { técnica a } \\
\text { escala }\end{array}$ & $\begin{array}{l}\text { Cambio en la } \\
\text { productividad } \\
\text { total de los } \\
\text { factores }\end{array}$ \\
\hline Arroz & 1.000 & 1.118 & 1.000 & 1.000 & 1.118 \\
Frijol & 1.004 & 1.115 & 1.000 & 1.004 & 1.120 \\
Maíz & 1.000 & 1.053 & 1.000 & 1.000 & 1.053 \\
\hline Promedio & 1.001 & 1.095 & 1.000 & 1.001 & 1.097 \\
\hline
\end{tabular}

TABLA 8

Promedio de los índices de Malmquist para los rubros arroz, frijoles y maíz. 1961 - 2014

Fuente Elaboración propia

Según estos datos los tres rubros experimentaron mejoría en el crecimiento promedio anual de la PTF. En el caso del rubro arroz el incremento en el índice de la PTF (11.8\%) se explica por un $11.8 \%$ de crecimiento promedio anual del cambio en la tecnología, es decir una mejora provocada por la utilización de mejores variedades de semilla.

Para el caso del rubro frijol, el cual experimento el mayor crecimiento promedio de la PTF (12\%) el índice se explica por un $11.5 \%$ de crecimiento de cambio en la tecnología y en menor medida por un $0.4 \%$ de cambio en la eficiencia técnica y en la eficiencia a escala respectivamente.

Para el rubro maíz el índice promedio de la PTF fuel menor en el período y de igual manera se explica a partir del crecimiento del cambio en la tecnología 5,3\%.

En el caso del comportamiento de los índices promedios de la PTF de los tres rubros, su crecimiento se explica a partir del cambio tecnológico, relacionado directamente con la incorporación y usos de mejores variedades de semilla a las cosechas, más que mejoras en la eficiencia técnica de la mano de obra.

Cabe destacar el hecho que durante el período en estudio el rubro que experimentó una mayor evolución en el crecimiento de las áreas de siembra fue el rubro del maíz, lo mismo que experimento el mayor crecimiento en sus volúmenes productivo, pero fue el de menor evolución en sus rendimientos por manzanas, lo que respalda ser el rubro con el menor crecimiento de su índice PTF. Y aclara el hecho de que el concepto de productividad no está relacionado directamente con el comportamiento de las áreas de siembras y de la obtención de volúmenes productivos. 
En cambio, el rubro arroz mostró la menor evolución en áreas de siembra durante el período, sin embargo obtuvo el mayor crecimiento en rendimientos productivos. (Ver gráficos 1,2 y 3 )

\begin{tabular}{lccc}
\hline Período & $\begin{array}{l}\text { Cambio en la } \\
\text { eficiencia Técnica }\end{array}$ & $\begin{array}{c}\text { Cambio en la } \\
\text { Tecnología }\end{array}$ & $\begin{array}{c}\text { Cambio promedio en la } \\
\text { PTF por décadas }\end{array}$ \\
\hline & & & \\
$1962-1971$ & 1.001 & 1.0606 & 1,037 \\
$1972-1981$ & 0.994 & 0.98 & 0,918 \\
$1982-1991$ & 0.986 & 0.839 & 0,825 \\
$1992-2001$ & 0.994 & 1.072 & 1.051 \\
$2002-2014$ & 1.013 & 0.889 & 0.902 \\
\hline
\end{tabular}

TABLA 9

Promedio de los índices de Malmquist para los rubros arroz, frijoles y maíz. Por Década. 1961 - 2014

Fuente Elaboración propia

La tabla No. 9 muestra los promedios de los índices Malmquist para cada rubro de grano básico en estudio, calculado por década. Se observa que el comportamiento de los índices PTF por década no es uniforme. De los cinco sub períodos estudiados en dos de ellos la PTF mostró un cambio positivo (1962-1971 y 1992-2001) y en los restantes un comportamiento negativo. Algunos estudios indican algunas causas históricas de carácter económico que explican el comportamiento de este indicador (PTF).

Bonilla Bird (2009) en su estudio "Análisis del sistema nacional de producción y certificación de semillas certificadas de granos básicos para la comercialización de semillas certificadas en los mercados locales y en Centroamérica" presenta algunos datos históricos que guardan relación con el comportamiento de los indicadores PTF en cada uno los sub períodos estudiados.

En la década 1962 - 1971 la PTF mostro un crecimiento promedio de 3.7\% explicado por un incremento del $6.0 \%$ del cambio tecnológico y apenas un $0.1 \%$ de mejoramiento dela eficiencia técnica. Según el estudio de Bonilla Bird pocos años antes de este período se había reglamentado la primera "Ley de semillas" (1959) lo que probablemente impulsó la utilización de semillas mejorada en los rubros de granos básicos.

En la década de los años 70 la producción y tenencia de semillas mejoradas se concentró en manos privadas y empresas transnacionales lo cual, si consideramos que la producción de granos básicos en Nicaragua ha estado mayoritariamente en manos de pequeños productores parceleros, redujo el acceso de estas tecnologías a los pequeños productores lo que incidió en un decrecimiento del índice PTF (-11.8\% respecto de la década anterior) para esa década, que también se caracterizó por una expansión dela Frontera agrícola, aumentando las áreas de siembra pero limitando el uso de nuevas tecnologías (semillas mejoradas). En esta década el Índice de cambio tecnológico decreció en un -8\%.

Par la década que inició en el 1980 la producción de semillas se concentró en manos del gobierno estableciendo una relación vertical Gobierno - productores para la entrega de semillas. Este sub período se caracterizó por el abandono de grandes extensiones de tierra ubicadas en zonas de guerra y lo mismo que grande cantidades de mano de obra fueron destinadas a participar en actividades de la guerra que caracterizó el período. Los niveles de inversión para el mejoramiento productivo fueron mínimos e insuficientes lo que se traduce en un indicador PTF aún menor y un decremento de un $-1.3 \%$ del indicador del cambio tecnológico respecto a la década anterior.

A inicios de los años 90 se presenta una desorganización completa para el sector semillero nacional por la reorganización del Estado. Se inicia un periodo posguerra afectando al sistema investigativo, transferencia, extensión y adopción de tecnología (Bonilla Bird 2009).

En la siguiente década (1992-2001) se dieron algunos cambios institucionales que propiciaron un crecimiento del indicador PTF del orden de un $7 \%$, y un crecimiento de $8 \%$, relacionados con la producción de semillas mejoradas, por ejemplo: 
- A mediados de los noventas se inició el Proyecto de Fortalecimiento de los servicios del Ministerio de Agricultura y Ganadería (FOSMAG/BID), con el cual se inician una serie de acciones para la consolidación y fortalecimiento de la Dirección General de Semillas, como uno de los componentes en la Dirección General de Sanidad Agropecuaria.

- Se dan las primeras iniciativas para la conformación de la Comisión Nacional de Semillas, conformada por todos los sectores involucrados en el sistema nacional de semillas en esa época. Se elaboró el marco regulatorio y normativo para la certificación de semillas, Ley 280 y su reglamento.

- Por medio de PROMESA se fomenta la comercialización y participación de las empresas productoras, comercializadoras, instituciones de gobierno, entes autónomos, Organismos no Gubernamentales, proyectos y agencias de cooperación internacional en la difusión de información y promoción del uso de semilla mejorada de manera masiva, esto incluye variedades de polinización libre e híbridos para el caso del cultivo del maíz, del uso de variedades mejoradas de frijol, sorgo y un poco en el cultivo del arroz, ya que este cultivo es promovido por la asociación de productores de arroz y el INTA por medio de la introducción de material genético procedente del CIAT.

En la última década estudiada también se suceden algunos hechos que pueden explicar la variación negativa del Indicador PTF (-6\%) sobre todo el bajo nivel de uso o cobertura de semilla mejorada en relación a las áreas de siembra de los diferentes rubros de granos básicos.

Para el caso del cultivo del frijol se observa una drástica no utilización de semilla certificada. De 350,000 manzanas sembradas en el territorio nacional durante todo el ciclo productivo del 2007-2008 al 2008-2009, solamente se logró tener una cobertura de 25,000 manzanas con semilla certificada (FUNICA 2012, MAGFOR/DGPSA2010).

Por último, señala Bonilla Bird, "en el cultivo del arroz se observa la misma tendencia, se incrementan las áreas de siembra estimulados por el precio del grano en el mercado local, las políticas proteccionistas hacia este sector de parte del gobierno controlando las importaciones de grano comercial, y por las características de polinización casi autógena de este cultivo. Todo esto permite que los arroceros no se motiven a la utilización de semilla certificada, pues usualmente la usan por varios ciclos hasta que los rendimientos se disminuyen dramáticamente por diferentes motivos, como es la disminución de calidad del grano por afectaciones de patógenos, mezcla varietal, infestación de campos con arroz rojo. De 120,000 manzanas a sembrarse en el ciclo 2008-2009, solamente se establecerán 19,000 manzanas con semilla certificada.

Los indicadores PTF obtenidos guardan alguna relación con los resultados de estudios similares que calcularon la PTF en el sector agrícola de nuestro país utilizando la misma metodología. Tabla 7

TABLA 7

Indicadores obtenidos en estudios previos sobre PTF en Nicaragua

\begin{tabular}{|c|c|c|c|c|c|c|}
\hline Autores & $\begin{array}{l}\text { Coelli and } \\
\text { Prasada }\end{array}$ & $\begin{array}{l}\text { Ludema } \\
\text { Carlos }\end{array}$ & Nin et al & $\begin{array}{l}\text { Ávila and } \\
\text { Evenson }\end{array}$ & $\begin{array}{l}\text { Trueblood } \\
\text { and } \\
\text { Coggins }\end{array}$ & Arnade \\
\hline $\begin{array}{l}\text { Fecha de } \\
\text { estudio }\end{array}$ & 2005 & 2010 & 2003 & 2004 & 2003 & 1998 \\
\hline $\begin{array}{l}\text { No. De } \\
\text { países }\end{array}$ & 93 & 120 & 115 & 82 & 115 & 70 \\
\hline Período & $1980-2000$ & $1961-2007$ & $1965-1994$ & $41961-2001$ & 1961-1994 & $41961-1993$ \\
\hline Método & DEA & DEA & DEA & OLS & DEA & DEA \\
\hline Nicaragua & 1,018 & 1,014 & 1,019 & 1,016 & 0,964 & 0,998 \\
\hline
\end{tabular}

Fuente: Zúniga-González 2011b

\section{Conclusiones}


El objetivo de este estudio ha estado centrado en medir el impacto de las tecnologías en la Productividad Total de los Factores de los cultivos de granos básicos Arroz, Frijol y Maíz durante el período 1961 - 2013 e identificar los factores de eficiencia e ineficiencia que han incidido en el comportamiento de este indicador. Los resultados indican la evolución del indicador PTF para cada rubro en particular y señalan la incidencia de los indicadores de Eficiencia Técnica y Cambio Tecnológico en la PTF de cada rubro.

Los promedios de los indicadores para el período en estudio en cada rubro, muestran una evolución positiva y evidencian una clara incidencia del indicador de Cambio tecnológico en la evolución del indicador PTF. Este cambio en la tecnología hace referencia a la utilización de mejores variedades de semillas en algunos sub períodos en estudio, lo que señala un derrotero a seguir por los diseñadores de políticas orientadas al sector agrícola, destacando la importancia de fortalecer el sistema de producción de semillas mejoradas y ampliar su nivel de cobertura a todos los sub sectores productivos.

Los indicadores obtenidos señalan también la poca incidencia de los cambios en la Eficiencia Técnica sobre la evolución del indicador PTF lo que urge diseñar programas orientados a mejorar las capacidades técnicas y las habilidades de gestión de los productores dedicados a la producción de estos rubros.

\section{ReFERENCIAS}

Arrimón Gabriel y Torrelo. Mariela. 1997. Productividad Total de los Factores: revisión metodológica y una aplicación al sector manufacturero uruguayo. LC/MVD/R.129.Rev2. CEPAL.

Aguilar, A. J., Urtecho, K. D. R. O., Olivares, M. D. S. P., González, C. A. Z., Palomares, R. D., Figueredo, P. S., \& Madrigal, O. J. Q. (2014). Efectos de la ineficiencia técnica ambiental en la calidad del agua del Estero Real: Caso Nicaragua. Universitas (León). Revista Científica de la UNAN-León., 5(2), 17-32.

Andrade Araujo, et al (2014). América Latina: productividad total de los factores y su descomposición. Revista CEPAL 114 - diciembre 2014.

Bravo-Ureta, B. E., Moreira, V. H., Arzubi, A. A., Schilder, E. D., Álvarez, J., \& Molina,

Bravo Uretra (2008b), Globalización y desarrollo económico: algunas consideraciones sobre la incidencia en el sector agrícola. Agro sur, Vol. 38.

Bonilla Bird. (2009) Análisis del sistema nacional de producción y certificación de semillas certificadas de granos básicos para la comercialización de semillas certificadas en los mercados locales y en Centroamérica. FUNICA. Mayo2009.

Caves D., Christensen L., Diewerte, (1982), "The Economic Theory of Index Numbers and the Measurement of Input, Output and Productivity”. Econométrica. Noviembre. 1393-414.

Coelli. T.J. (2008). “A guide to DEAP, version 2:1 A data envelopment analysis program.” CEPA working papers, Departments Econometrics, University of New England. Australia.

DGPSA-MAGFOR. (2010). Dirección General de Semillas. Programa Nacional de Semillas de Granos Básicos y Oleaginosas.

FUNICA (2012). "Estado actual y propuesta de acción del sector agropecuario y forestal en Nicaragua, 1ra Ed. ISBN 978-99964-827-2-4.

Lanteri L. N. (2002). "Crecimiento, Términos de Intercambio Externos y Productividad Total de los Factores: evidencia para la economía Argentina, 1955-2001”. Mimeo.

Lanteri, L. N. (2002). Productividad, desarrollo tecnológico y eficiencia. La propuesta de los índices Malmquist. In anales de la asociación argentina de Economía política.

Martínez-Damián Miguel A., José J. Bambilla-Paz, Roberto García-Mata (2013). Agricultura sociedad y desarrollo, vol.10 no.3 Texcoco jul./sep. 2013.

Rutan, V. (2002). Productivity Growth in World Agriculture: Sources and Constraints. Journal of Economic Perspectives 16:161-184. 
Reyez Bernal, José (2010). "El residuo de Solow revisado" Revista de Economía Institucional, vol. 12, n. ${ }^{\circ} 23$, segundo semestre/2010, pp. 347-361.

Lema y Brescia. (2001) Medición del cambio tecnológico, la productividad y la eficiencia en el sector agropecuario. Buenos Aires, 2001.

Ludema, Carlos E. (2010). Agricultural productivity growth, efficiency change and technical progress in Latin America and the Caribbean. BID. 2010

Sotelsek y Laborda. (2010). América Latina: medición de la eficiencia productiva y el cambio técnico incorporando factores ambientales. Revista c e p a 1101 . Agosto 2010.

Trueblood, M.A. and Coggins, J. (2003), Intercountry Agricultural Efficiency and Productivity: A Malmquist Index Approach,, mimeo, World Bank, Washington D.C

Zúniga-González, Carlos A. (2011a). Impacto de la política salarial y de empleo en la productividad de los sectores productivos en Nicaragua, 1994-2010. Agecon Search http://purl.umn.edu/118454

Zúniga-González Carlos Alberto y Navarrete Blanco, Ángel (2011b). Impacto de la tecnología INTA Chinandega en la productividad de las fincas del Occidente de Nicaragua, 2003-2009. Agecon Search http:// purl.umn.edu/109312

Zúniga-González., Carlos, A. (2011c), Technical efficiency of organic fertilizer in small farm of Nicaragua. 1998-2005. African Journal of Business Management Vol. 5 (3). pp 967-973, 4 February, 2011. DOI:10.5897/AJBM10.873

Saini, E., \& Lema, D. (2015). Agricultural Productivity in Argentina (No. 1008-2016-79825).

FÄRE, R., GROSSKOPF, S., \& LINDGREN, B. Y ROOS, P.[1989]: Productivity Developments in Swedish. Mimeo. Olavarría Gambi, M. (2005). Acceso a la salud en Chile. Acta bioethica, 11(1), 47-64.

Araujo, J. A. D., Feitosa, D. G., \& Silva, A. B. D. (2014). Latin America: Total factor productivity and its components.

Lema, R. D., Gatti, N., \& Brescia, V. (2015). A meta-frontier approach to measuring technical efficiency and technology gaps in beef cattle production in Argentina.

Shephard, R.W., (1970), Theory of cost and production functions (Princeton University Press, Princeton).

Jordan, C. F., \& Russell, C. E. (1983). Jari: Productividad de las plantaciones y perdida de nutrientes debido al corte y la quema. Interciência, 8(6), 294-297.

Runge, C. F., \& Senauer, B. (2007). How biofuels could starve the poor. Foreign Aff., 86, 41.

Von Braun, J. (2007). The world food situtation: new driving forces and required actions. Intl Food Policy Res Inst.

Solow, Robert M. (1957). Technical Cahnge and the Aggregate Production Function, Review of Economics and Statistic, 39 August, 312-320

Coelli, T. J., Prasada Rao, D. S., O’Donnell, C. J., \& Battese, G. E. (2005). Additional topics on data envelopment analysis. An introduction to efficiency and productivity analysis, 183-208.

Nin, A., Arndt, C., \& Preckel, P. V. (2003). Is agricultural productivity in developing countries really shrinking? New evidence using a modified nonparametric approach. Journal of Development Economics, 71(2), 395-415.

Martin, W., and Mitra, D. (1999). Productivity Growth and Convergence in Agriculture and Manufacturing. World Bank Working Paper Number 2171, World Bank, Washington DC.

Arnade, C. (1998). Using a programming approach to measure international agricultural efficiency and productivity. Journal of Agricultural Economics, 49(1), 67-84.

Fulginiti, L. E., \& Perrin, R. K. (1997). LDC agriculture: Nonparametric Malmquist productivity indexes. Journal of development economics, 53(2), 373-390.

Lema, D. (1999). Crecimiento y Productividad de la Agricultura Argentina. Instituto de Economía y Sociología RuralINTA.

Nin-Pratt, A., Falconi, C., Ludena, C. E., \& Martel, P. (2015). Productivity and the performance of agriculture in Latin America and the Caribbean: from the lost decade to the commodity boom (No. IDB-WP-608). IDB Working Paper Series. 
Fulginiti, L. and R.K. Perrin. 1993. Prices and Productivity in Agriculture. Review of Economics and Statistics, 75(3) 471-482.

Weibe, K., M. Soule, C. Narrod, and V. Breneman. (2000). Resource Quality and Agricultural Production: A Multicountry Comparison, mimeo, US Department of Agriculture, Economic Research Service, Washington, D.C., July 2000.

Bravo-Ortega, C. and D. Lederman, (2004). Agricultural Productivity and Its Determinants: Revisiting International Experiences. Estudios de Economia, 31(2), 133-163. Universidad de Chile, Santiago de Chile.

Ludena, C.E., T.W. Hertel, P.V. Preckel, and A. Nin. 2007. Productivity Growth and Convergence in Crop, Ruminant and Non-Ruminant Production: Measurement and Forecasts, Agricultural Economics, 37(2007): 1-17.

Nin Alejandro and Yu Bingxin (2008), Developin Countries and Total Factor Productivity Growth in Agriculture: New Evidences Using a Malmquist Index with Constraines Implicit Shadow Prices

Coelli TJ, y Prasada Rao D.S (2003), Total factor productivity growth in agriculture: a Malmquist index analysis of 93 countries, 1980-2000. International Association of Agricultural Economics. Durban, August 2003.

Avila, A.F.D., and R.E. Evenson (2005). Total Factor Productivity Growth in Agriculture: The Role of Technological Capital, In Volume 3B of the Handbook of Agricultural Economics, B.L. Gardner and G.C. Rausser eds. Amsterdam; New York: Elsevier.

Fare, R., S. Grosskopf, M. Norris and Z. Zhang (1994), Productivity Growth, Technical Progress, and Efficiency Changes in industrialized Countries, American Economic Review, 84, 66-83.

Ludena, C.E., T.W. Hertel, P.V. Preckel, K. Foster, and A. Nin. (2005). Cambios Tecnológicos y Productividad en el Sector Agropecuario: Un Análisis de la Comunidad Andina, Cuestiones Económicas, 21(1): 61-84.

Banco Central de Nicaragua (2009). Informes Estadisticos Economicas 1961-2004. 